\title{
A Formal description for robots understanding knowledge
}

\author{
Yuanxiu Liao ${ }^{1,2, a}$ Shengming Zhou ${ }^{1,2, b, *}$ Zhi Li ${ }^{1,2, c}$ \\ ${ }^{1}$ Guangxi Key Lab of Multi-source Information Mining \& Security, Guangxi Normal University, Guilin \\ 541004, China \\ ${ }^{2}$ College of Computer Science \& Information Engineering, Guangxi Normal University, Guilin \\ 541004, China
}

aliaoyuanxiu@gxnu.edu.cn, bsmzhou@gxnu.edu.cn, czhili@gxnu.edu.cn

\begin{abstract}
Keywords: knowledge understanding; autonomous knowledge; formal description; semantic interpretation; logical framework

Abstract. In the research field of robotics, as can be seen from the description of the classification of the robot, the autonomous robots should have their own knowledge. Generally, a robot's knowledge refers to the knowledge in the knowledge base of the robot systems. However, Can a robot understand its knowledge? How describes it? In this study we present a logical framework used to describe robots understanding knowledge. Our framework is a first logic system with a semantic interpretation based on sensed data. In this framework, we give a formal definition that a robot understands knowledge, and we define autonomous knowledge of the robot, i.e. the knowledge belonging to the robot itself. More specifically, if a robot has the ability to acquire knowledge through its sensors and can understand, and apply this knowledge in performing tasks, then this knowledge is considered to be the robot's autonomous knowledge. The goal of our work is to formalize these performances of the robot systems. The robots that is described in the proposed framework would have much highly autonomy in the real world.
\end{abstract}

\section{Introduction}

In recent years, improving the understanding ability of intelligent system has become a hot research topic. A variety of automatic understanding technology has been highly concerned, such as image understanding, natural language processing, human behavior understanding, visual semantic understanding in big data, the surrounding environment understanding in reasoning about action, semantic and context understanding in Web search etc. The latest research results include the following literatures. Klaus Broelemann etc[1] presented a new approach for novel sketch map understanding in an offline way to deal with the major problems of sketch map understanding, such as vagueness in interpretation. They discussed a region-growing segmentation for sketch map objects, a classification for isolated objects, and a context-aware classification. The context-aware classification uses probabilistic relaxation labeling to integrate dependencies between objects into the recognition. Liang $\mathrm{Li}$ etc[2] studied distributed image understanding with semantic dictionary and semantic expansion, and proposed a semantic dictionary to describe the images on the level of semantic, used to solve the problem of visual polysemia and concept polymorphism in the image understanding. The distributed framework on the basis of the semantic dictionary aims to speed up the large scale image understanding. Irfanullah etc[3] explored adding semantics to the reliable object annotated image databases, and proposed semantically enhanced information extraction model that calculate the semantic intensity of each object in the image and then enhance the tagged concept with the assistance of lexical and conceptual knowledge bases. Then noises, redundant and unusual words are filtered out by means of various techniques, such as semantic similarity, stopwords and words unification etc. A. Terai and M. Nakagawa[4] constructed a computational model of metaphor understanding based on statistical corpora analysis. Their model includes a categorization process and a dynamic-interaction process, which is realized based on a recurrent neural network. The proposed model is able to highlight the emphasized features of a metaphorical expression. Eriko Yoshimura etc[5] study the issue on comprehending the significance of simile expressions by using an intuitive sensory association method implemented on computer. The sensory association method outputs the sensations and impressions that humans naturally feel in 
response to a given noun, and the associations between words are realized by an association mechanism using a concept base, a large-scale knowledge base. the goal of the proposed system is to teach a computer to understand similes. Rishiraj Saha Roy etc[6] studied discovering and understanding word level user intent in Web search queries. They proposed that words in queries can be classified as either content or intent, where content words represent the central topic of the query, while users add intent words to make their requirements more explicit. This work focus on intent word discovery and understanding. They argue that intelligent processing of intent words can be vital to improving the result quality. Zheng Zhou etc[7] designed a deep image understanding framework to implement a description generator for general images involving human activities. They proposed a deep hierarchical model to realize the image recognition and a syntactic tree-based model to realize the natural language generation. The proposed framework is able to recognize the human-object interaction activity in the image based on the co-occurrence analysis of 3-D spatial layout and generate natural language description according to what is really happening in the image. Guglielmo Gemignania etc[8] investigated interactive environmental knowledge acquisition, and proposed a novel approach to semantic mapping. Instead of requiring robots to autonomously learn every possible aspect of the environment, the authors proposed allowing non-expert users to shape robot knowledge through human-robot interaction. Thus, they present a novel representation of the environment that combines the metric information needed for navigation tasks with the symbolic information that conveys meaning to the elements of the environment and the objects therein. In this way, multiple AI techniques are exploited to solve spatial referring expressions and support task execution. Alex Doboli etc[9] investigated modeling semantic knowledge structures for creative problem solving, and presented a model for semantic network representation. The proposed model can help to correctly and quickly understand the features and structure of the solutions, and then make reliable predictions about their outcomes and rewards. Weihua Sheng etc[10] proposed a new robot semantic mapping approach through human activity recognition in a human-robot coexisting environment. This approach offers a new perspective for robot semantic mapping and can significantly reduce the difficulties involved in traditional vision-based object classification algorithms. Semantic information is able to help robots understand unknown environments better. KarinneRamirez-Amaro etc[11] studied transferring skills to humanoid robots by extracting semantic representations from observations of human activities, and presented a method that allows robots to obtain and determine a higher-level understanding of a demonstrator's behavior via semantic representations. Thus, a meaningful semantic description is obtained in terms of human motions and object properties. This abstraction from observations captures the "essence" of the activity, thereby indicating which aspect of the demonstrator's actions should be executed in order to accomplish the required activity. Hamdi Aloulou etc[12] studied handling uncertainty in sensorbased context aware applications, and proposed a method for the measurement of uncertainty based on both physical and operational behaviors of the sensors. The approach of uncertainty handling in context aware reasoning allowed the retro-compatibility with naive classical semantic reasoning where information from the real world are perceived as absolute truth and decisions are considered certain based on these information. This paper describes how the level of uncertainty is incorporated into different layers of a semantically driven context aware system and how it is transferred to a decision engine in order to perform more accurate decisions in ambiguous observations.

Most of the existing understanding models are based on statistical techniques or other computational methods. For example, The metaphor understanding model in [4] is constructed based on the results of the statistical language analysis. The model FTWIM in [7] can be regard the as an energy-based model in statistic thermodynamics and determine the parameters of the model using a mathematical optimization method. In the sensory association method[5], associations between words are realized by an association mechanism using a concept base, a large-scale knowledge base. And the candidate sensory words are selected via the statistical results of 7 operations. The main tasks of the information extraction model in [3] is to calculate the semantic intensity of each object in the image and then enhance the tagged concept with the assistance of 
lexical and conceptual knowledge bases. Automatic understanding of sketch maps in [1] is a method based on a context-aware classification, where the context-aware classification uses probabilistic relaxation labeling to integrate dependencies between objects into the recognition. Discovering and understanding word level user intent in Web search queries in [6] uses Cooccurrence statistics as function word indicators. The semantic dictionary in [2] characterizes the probability distribution between visual appearances and semantic concepts, and the learning procedure of semantic dictionary is formulated into a minimization optimization problem. Robot semantic mapping through human activity recognition[10] uses a wearable sensing and computing approach.

However, the methods based on statistical techniques or other computational methods have limitations in understanding concept and knowledge. It is difficult to understand the meaning of a concept or knowledge by calculating. We know that logical frameworks typically have strong representation and reasoning abilities. In this study we present a logical framework used to describe robots understanding knowledge. In the proposed framework, we give a formal definition that a robot understands knowledge, and we define autonomous knowledge of robots, i.e. the knowledge belonging to the robot itself.

\section{A Logical Framework for Describing Robots’ Knowledge}

This section introduces a knowledge representation for robots. In our discussion, we assume that the robots are equipped with sensors and information processing units, which can recognize the surrounding environment.

The Logical Framework $\mathbf{L}_{\mathbf{R K}}$ - we build a logical framework $L_{R K}$ for describing robots' knowledge. $\mathrm{L}_{\mathrm{RK}}$ is an expansion of the traditional situation calculus action theory by adding two kinds of sensors and defining a novel semantics based on sensing data. Most concepts and symbols concerned action theories and situation calculus in this paper can see [13].

The syntax of $\mathrm{L}_{\mathrm{RK}}$ is constructed as follows:

The alphabet in $\mathrm{L}_{\mathrm{RK}}$ :

- The alphabet of the standard first order logic;

- Individual variable symbols for action: $a_{1}, a_{2}, \cdots$;

- Individual variable symbols for situation: $s_{1}, s_{2}, \cdots$;

- Individual variable symbols for object: any usable symbol.

- Finite specific functional constant symbols: $\boldsymbol{f}, \boldsymbol{g}, \boldsymbol{h}, \cdots$, these specific symbols correspond to the functional sensors fixed on robots.

- Finite specific predicate constant symbols: $\boldsymbol{P}, \boldsymbol{Q}, \boldsymbol{R}, \cdots$, these specific symbols correspond to the relational sensors fixed on robots.

The formation rules of terms and well formula are the same as in the standard first order logic and the traditional situation calculus.

Sensors and Sensing Actions in $\mathrm{L}_{\mathrm{RK}}$. The sensors here are mechanical settings fixed on robots. Each sensor has a specific function. For example, they can independently identify a desk, a book, or the position relationship between two objects, and so on. Sensors are denoted by $\overline{B O O K}(\mathrm{x})$, $\overline{D E S K}(\mathrm{x}), \overline{O N}(\mathrm{x}, \mathrm{y}), \cdots$, their functions are to check whether certain objects have a particular relationship. The output values of these sensors are "YES", or "NO".

Sensing actions are those robots perform using their sensors to get external environment information. For example, When a robot wants to check whether the object $a$ is a book, the robot needs to perform a sensing action: starts the sensor $\overline{B O O K}(\mathrm{x})$ and use $\overline{B O O K}(\mathrm{x})$ to work on $a$. If $a$ is a book, then the output value of $\overline{B O O K}(\mathrm{a})$ is "YES"; if $a$ is not a book, then the output value of $\overline{B O O K}(\mathrm{a})$ is "NO".

The Semantics of $L_{R K}$. The semantic interpretations of the formulas in $L_{M K}$ are defined as follows: 
- Let $\mathbf{P}\left(\mathrm{x}_{1}, \mathrm{x}_{2}, \cdots, \mathrm{x}_{\mathrm{n}}\right)$ be a specific atomic predicate formula in $\mathrm{L}_{\mathrm{RK}}, \quad \bar{P}\left(\mathrm{x}_{1}, \mathrm{x}_{2}, \cdots, \mathrm{x}_{\mathrm{n}}\right)$ be its corresponding sensor, and $\mathrm{a}_{1}, \mathrm{a}_{2}, \cdots, \mathrm{a}_{\mathrm{n}}$ be individual objects in the robot's environment, then

$\mathbf{P}\left(\mathrm{a}_{1}, \mathrm{a}_{2}, \cdots, \mathrm{a}_{\mathrm{n}}\right)$ is true, if the output value of $\bar{P}\left(\mathrm{a}_{1}, \mathrm{a}_{2}, \cdots, \mathrm{a}_{\mathrm{n}}\right)$ is "YES"; $\mathbf{P}\left(\mathrm{a}_{1}, \mathrm{a}_{2}, \cdots, \mathrm{a}_{\mathrm{n}}\right)$ is false, if the output value of $\bar{P}\left(\mathrm{a}_{1}, \mathrm{a}_{2}, \cdots, \mathrm{a}_{\mathrm{n}}\right)$ is "NO".

- The interpretations for $\neg P\left(a_{1}, a_{2}, \cdots, a_{n}\right), \quad P\left(a_{1}, a_{2}, \cdots, a_{n}\right) \wedge Q\left(a_{1}, a_{2}, \cdots, a_{n}\right)$, $P\left(a_{1}, a_{2}, \cdots, a_{n}\right) \vee Q\left(a_{1}, a_{2}, \cdots, a_{n}\right)$, and $P\left(a_{1}, a_{2}, \cdots, a_{n}\right) \rightarrow Q\left(a_{1}, a_{2}, \cdots, a_{n}\right)$ are as usual.

- $\quad \exists x P(x)$ is true iff for some object a in the robot's environment, $\mathrm{P}(\mathrm{a})$ is true.

- $\forall \mathrm{xP}(\mathrm{x})$ is true iff all object a in the robot's environment, $\mathrm{P}(\mathrm{a})$ is true.

It is easy to prove following theorem from the Semantics of $\mathrm{L}_{\mathrm{RK}}$.

Theorem 1 Let $\mathbf{P}\left(\mathrm{x}_{1}, \mathrm{x}_{2}, \cdots, \mathrm{x}_{\mathrm{n}}\right)$ be a specific atomic predicate formula in $\mathrm{L}_{\mathrm{RK}}$, and $\mathrm{a}_{1}, \mathrm{a}_{2}$, $\cdots, a_{n}$ be individual objects in the robot's environment, then the robot can indentify whether $\mathbf{P}\left(\mathrm{a}_{1}, \mathrm{a}_{2}, \cdots, \mathrm{a}_{\mathrm{n}}\right)$ is true or not.

\section{The Model for Robots Understanding Knowledge}

In this section, we construct a model for robots understanding knowledge and define robots' autonomous knowledge. For the purposes of discussion, we introduce an operator "Und" in $\mathrm{L}_{\mathrm{RK}}$. $\operatorname{Und}(\mathrm{R}, \varphi)$ denotes " $\mathrm{R}$ understands $\varphi$ ", where $\mathrm{R}$ is a robot, $\varphi$ is a formula in $\mathrm{L}_{\mathrm{RK}}$. Und $(\mathrm{R}, \varphi)$ as a formula is added to $\mathrm{L}_{\mathrm{RK}}$.

Definition 1 A knowledge base is a collection of some sentences in $L_{M K}$. For a robot $R$, the knowledge base of the robot $\mathrm{R}$ is denoted as KB-R.

Definition 2 Let $\mathrm{P}(\mathrm{x})$ be a unary atomic predicate formula representing a concept $\mathrm{P}$, Ont $(\mathrm{P})$ be an set of sentences used to describe the ontology of $P$ in $L_{M K}$, then $\operatorname{Ont}(P)$ is referred to as the ontology of $\mathrm{P}$. The ontology base of a robot $\mathrm{R}$ in $\mathrm{L}_{\mathrm{MK}}$ is denoted as Ont-R.

Definition 3 Let $\mathrm{P}(\mathrm{x})$ be a unary atomic predicate formula representing a concept $\mathrm{P}, \mathrm{Kno}(\mathrm{P})$ be an set of sentences used to describe the knowledge about $\mathrm{P}$ in $\mathrm{L}_{\mathrm{MK}}$, then $\mathrm{Kno}(\mathrm{P})$ is referred to as the knowledge about $\mathrm{P}$.

Definition 4 (basic formulas) Let $\mathrm{BF}=\left\{\varphi \mid \varphi\right.$ is formed by specific predicate in $\left.\mathrm{L}_{\mathrm{MK}}\right\}$, then $\mathrm{BF}$ is referred to as the basic formulas of $\mathrm{L}_{\mathrm{MK}}$.

Definition 5 Let $\mathrm{P}(\mathrm{x})$ be a unary predicate that represents an concept $\mathrm{P}$ in $\mathrm{L}_{\mathrm{MK}}$, $\mathrm{R}$ be a robot, we say that $\mathrm{R}$ understands $\mathrm{P}$, denoted as $\operatorname{Und}(\mathrm{R}, \mathrm{P})$, if the following conditions are satisfied:

- for any individual object a in R's environment, R can identify whether $\mathrm{P}(\mathrm{a})$ is true;

- the ontology base of $\mathrm{R}$ includes the ontology that describes the concept $\mathrm{P}$;

- the knowledge base of $\mathrm{R}$ includes the knowledge about $\mathrm{P}$;

Definition 6 A model of the robot understanding knowledge, referred to simply as "understanding model", is a triples $\mathrm{M}=<\mathrm{B}, \mathrm{O}-\mathrm{R}, \mathrm{K}-\mathrm{R}>$, where $\mathrm{B} \subseteq \mathrm{BF}$ is a subset of the basic formulas of $\mathrm{L}_{\mathrm{MK}}, \mathrm{O}-\mathrm{R} \subseteq$ Ont- $\mathrm{R}$ is a set of the ontology base of a robot $\mathrm{R}$ in $\mathrm{L}_{\mathrm{MK}}$, K-R $\subseteq K B-\mathrm{R}$ is a set of the knowledge base of the robot $\mathrm{R}$.

Theorem 2 Let $\mathrm{P}(\mathrm{x})$ be a unary predicate that represents an concept $\mathrm{P}$ in $\mathrm{L}_{\mathrm{MK}}, \mathrm{M}=<\mathrm{B}, \mathrm{O}-\mathrm{R}, \mathrm{K}$ $\mathrm{R}>$ be a understanding model, then $\operatorname{Und}(\mathrm{R}, \mathrm{P})$ is true in $\mathrm{M}$ if and only if $\mathrm{P}(\mathrm{x}) \in \mathrm{B}$, Ont $(\mathrm{P}) \subseteq \mathrm{O}-\mathrm{R}$, and $\mathrm{Kno}(\mathrm{P}) \subseteq \mathrm{K}-\mathrm{R}$.

$\mathrm{Und}(\mathrm{R}, \mathrm{P})$ is true in $\mathrm{M}$ is also denoted as $\mathrm{M} \vDash \mathrm{Und}(\mathrm{R}, \mathrm{P})$. Thus theorem 1 can represent as: $\mathrm{M} \vDash \operatorname{Und}(\mathrm{R}, \mathrm{P})$ iff $\mathrm{P}(\mathrm{x}) \in \mathrm{B} \wedge \mathrm{Ont}(\mathrm{P}) \subseteq \mathrm{O}-\mathrm{R} \wedge \mathrm{Kno}(\mathrm{P}) \subseteq \mathrm{K}-\mathrm{R}$.

\section{Conclusions}

A robot's intelligence level depends on its knowledge and the ability to apply knowledge. We have been introduced a logical framework for describing robots' knowledge and a model for robots understanding knowledge. We focus on three aspects of characteristics in the definition of understanding a concept. (1) the robot should identify the instance that the concept describes; (2) the robot should know the attributes of the concept; (3) the robot should have the ability to apply 
knowledge. This is the reason that we use a triples $\mathrm{M}=<\mathrm{B}, \mathrm{O}-\mathrm{R}, \mathrm{K}-\mathrm{R}>$ to construct the understanding model.

In this paper we only present a model for robots understanding concepts. In order to make the autonomous robots understanding more complex knowledge, it is needed to expand Und(R, $\varphi$ ) to a more general form, i.e. $\varphi$ is a general formula in $\mathrm{L}_{\mathrm{MK}}$. In other hand, the ontology base and the knowledge base is more abundant, the robots' understanding ability is stronger. Our further work is to research more perfect models for robots understanding knowledge.

\section{Acknowledgments}

This work was supported by the National Natural Science Foundation of China (Grant Nos. 61262005, 61262004) and Guangxi Natural Science Foundation (Grant No. 2013GXNSFAA019345).

\section{References}

[1] K. Broelemann, X. Jiang, A. Expert Systems With Applications, 45 (2016) 195-207

[2] L. Li, C. C. Yan, X. Chen, etc. Neurocomputing, 174(2016)384-392

[3] Irfanullah, N. Aslam, J. Loo, etc. Adding semantics to the reliable object annotated image databases[J]. Procedia Computer Science, 3 (2011) 414 - 419

[4] A. Terai, M. Nakagawa. Cognitive Systems Research, 19 - 20 (2012) 30 - 38

[5] E. Yoshimura, M. Imono, S. Tsuchiya, etc. Procedia Computer Science, 60 ( 2015 ) 55 - 62

[6] R. S. Roy, R. Katare, N. Ganguly etc. Web Semantics: Science, Services and Agents on the World Wide Web, 30 (2015) 22-38

[7] Z. Zhou, K. Li, L. Bai. Neural Comput \& Applic, DOI 10.1007/s00521-015-2171-x

[8] G. Gemignani, R. Capobianco, E. Bastianelli, etc. Robotics and Autonomous Systems (2016), http://dx.doi.org/10.1016/j.robot.2015.11.001

[9] A. Doboli, A. Umbarkar, S. Doboli, etc. Knowledge-Based Systems, 78 (2015) 34-50

[10] W. Sheng, J. Du, Q. Cheng etc. Robotics and Autonomous Systems, 68 (2015) 47 - 58

[11] K. Ramirez-Amaro, M. Beetz, G. Cheng. Artificial Intelligence (2015), http://dx.doi.org/10.1016/j.artint.2015.08.009

[12] H. Aloulou, M. Mokhtari, T. Tiberghien etc. Knowledge-Based Systems, 77 (2015) 16 - 28

[13] R. Reiter. Knowledge in Action: Logical Foundations for Specifying and Implementing Dynamical Systems, MIT Press, Cambridge, MA, 2001. 